\title{
Comparative analysis of single cell lung atlas of bat, cat, tiger and
}

\section{pangolin}

Xiran Wang ${ }^{1,2, \dagger}$, Zhihua $\mathrm{Ou}^{3,4, \dagger}$, Peiwen Ding ${ }^{3,5, \dagger}$, Chengcheng Sun ${ }^{3,5,6}$, Daxi Wang ${ }^{3,4}$,

Jiacheng $\mathrm{Zhu}^{3,5}$, Wendi $\mathrm{Wu}^{3,5,6}$, Yanan Wei ${ }^{3,5,6}$, Xiangning Ding ${ }^{3,5}$, Lihua Luo ${ }^{3,5}$, Meiling $\mathrm{Li}^{3}$, Wensheng Zhang ${ }^{7}$, Xin $\mathrm{Jin}^{3}$, Jian Sun ${ }^{1,2,}$, Huan $\mathrm{Liu}^{3,5, *}$ Dongsheng Chen $^{3,5, *}$

1. National Risk Assessment Laboratory for Antimicrobial Resistance of Animal Original Bacteria, South China Agricultural University, Guangzhou, China

2. Guangdong Laboratory for Lingnan Modern Agriculture, Guangzhou, China

3. BGI-Shenzhen, Shenzhen 518083, China

4. Shenzhen Key Laboratory of Unknown Pathogen Identification, BGI-Shenzhen, Shenzhen 518083, China.

5. College of Life Sciences, University of Chinese Academy of Sciences, Beijing 100049, China

6. School of Basic Medicine, Qingdao University, Qingdao 266071, China

7. School of Basic Medical Sciences, Binzhou Medical University, No. 346, Guanhai Road, Laishan District, Yantai City, Shandong, China

${ }^{\dagger}$ These authors contributed equally

* Correspondence should be addressed to Dongsheng Chen (chendongsheng@genomics.cn), Huan Liu (liuhuan@genomics.cn) and Jian Sun (jiansun@scau.edu.cn) 


\section{ABSTRACT}

Horseshoe bats (Rhinolophus sinicus) might help maintain coronaviruses severely affecting human health, such as SARS-CoV and SARS-CoV-2. It has long been suggested that bats may be more tolerant of viral infection than other mammals due to their unique immune system, but the exact mechanism remains to be fully explored. During the COVID-19 pandemic, multiple animal species were diseased by SARS-CoV-2 infection, especially in the respiratory system. Herein, single-cell transcriptomic data of the lungs of a horseshoe bat, a cat, a tiger, and a pangolin were generated. The receptor distribution of twenty-eight respiratory viruses belonging to fourteen viral families were characterized for the four species. Comparison on the immune-related transcripts further revealed limited cytokine activations in bats, which might explain the reason why bats experienced only mild diseases or even no symptoms upon virus infection. Our findings might increase our understanding of the immune background of horseshoe bats and their insensitivity to virus infections.

Bats are important reservoir hosts for a myriad of viruses including coronaviruses, rabies viruses, Hendra viruses, influenza viruses, etc. They were mostly asymptomatic or only developed mild diseases during viral infections by Ebola viruses, coronaviruses, henipaviruses, etc. The immune response induced by virus infection was shown to differ between human and bat cells ${ }^{1,2}$ and that bats may have their unique transcripts that are not present in other mammals ${ }^{3}$ Bats were found to have limited interferon activation due to mutation in the STING protein $^{4}$ and have contracted type I IFN $\alpha$ locus but constitutive IFN $\alpha$ expression without viral stimulation ${ }^{5}$. The unique immune response pathways and antiviral gene expression profile of bats, may promote their tolerance to viral infections ${ }^{6}$. Bats are probably the initial host of SARS-CoV-2 $2^{7,8}$, the etiological virus causing COVID-19. Although the direct progenitor of SARS-CoV-2 remains unknown, its closest relative (RaTG13) has been detected in a horseshoe bat (Rhinolophus sinicus), indicating horseshoe bats as its potential reservoir hosts. Moreover, horseshoe bats (genus Rhinolophus) were also found to harbor other groups 
of coronaviruses including the SARS-CoV emerged in China from 2002 to $2003^{9-11}$, indicating their critical role in the maintenance of human sensitive coronaviruses. Since the outbreak of COVID-19, multiple animal species have been infected and diseased by SARS-CoV-2, including pangolins, cats, tigers, etc. ${ }^{12-16}$. Herein, we conducted a comparative study using single cell transcriptomic data to elucidate the lung immune landscape of bat, cat, tiger and pangolin, which might help reveal the molecular basis for their differential immune behaviors upon infections by SARS-CoV-2.

Due to species-specific immune response upon viral infection, clinical symptoms in the lower respiratory differ among species. While bats, cats, tigers and pangolins were all permissive to SARS-CoV-2 infection, details of their biological background remain unknown. Herein, we collected lung tissues from healthy individuals of bats to generate single-nucleus libraries of lung cells, resulting in a total of 11838 pulmonary cells passing quality control (Figures $\mathbf{1 A}$ and 1B). Nine major cell types were identified in the lung atlas of bats, which included alveolar type 1 cells (AT1), alveolar type 2 cells (AT2), ciliated cells, secretory cells, endothelial cells, fibroblasts, T cells, B cells and macrophages, each demonstrating the specific expression of canonical cell type markers (Figure 1C, Table S1).

Because receptor binding is critical for viral entry into cells and that the distribution of receptors reveals the susceptibility of cells to viral infection, which may further stimulate the local immune response. Therefore, we determined the expression patterns of 29 genes encoding entry receptors of respiratory viruses in the lung cells of the four species. Compared with the other three species, Itgb5 (a receptor of adenoviruses) and Anpep (a receptor of human coronavirus 229E) were enriched in bat AT1 and AT2, respectively. Another adenovirus receptor, Cd86, was enriched in the macrophages of bat, pangolin and tiger. The rhinovirus receptor, $C d h r 3$, was enriched in the ciliated cells of both bat and cat. Adeno-associated virus receptor Rpsa were significantly expressed in tiger pulmonary cells except unannotated $\mathrm{T}$ and $\mathrm{B}$ cells. $A C E 2$, receptor for SARS-CoV and SARS-CoV-2, were relatively highly expressed in ciliated and secretory cells of cat and in the secretory cells of tiger. Only marginal expressions of ACE2 were observed in bat ciliated cells. However, the receptor for 
SARS-CoV-2, Scarb1, displayed highly specific expression in bat endothelial cells and macrophages. Another two SARS-CoV-2 receptors, Nrpl and Axl, also showed significant cell type and species specificity. Nrpl was largely enriched in AT1/AT2 of tigers and AT2 of bats, whereas Axl was highly expressed in fibroblasts and macrophages of bat lung, and fibroblasts of pangolin lung (Figure 1D).

Cytokine storm, due to uncontrolled and excessive release of pro-inflammatory cytokines, is one of the main culprits contributing to severe lung pathogenesis caused by various virus infections ${ }^{17}$. As a natural reservoir for zoonotic viruses, bats display no significant symptoms after virus infection thanks to its unique immunity ${ }^{18}$. Here, we compared the expression profiles of a variety of pro-inflammatory cytokines (IL1, IL6, TNF, interferons) and anti-inflammation cytokines (IL10, TGF beta) among distinct pulmonary cell types of bat, cat, pangolin, and tiger. Regarding anti-inflammation cytokines and corresponding receptors, no significant differences were observed among the pulmonary cells of the four species (Figure S1). However, receptors for specific pro-inflammation cytokines, IL-6 (Osmr, Lifr) and interferons (IFN) (Ifngr2, Ifnar1, Ifnar2), were significantly lowly expressed in bat pulmonary cells (Figure S1). Lif was suggested to be a mediator of pro-inflammation in several inflammatory disorders ${ }^{19}$ and was lowly expressed in bat. Accumulating evidence has indicated that $O S M$ mediates lung inflammations ${ }^{20}$ and that $O s m r$ was abundantly expressed on mice pulmonary endothelial and fibroblast cells. Similarly, we observed enrichment of Osmr in these two cell types of cat and tiger, and wide expressions in all the nine pulmonary cell types of pangolin. Interestingly, expression of Osmr was almost lost in bat lung cells. Type 1 interferons (IFNs) play central roles in initiating lung inflammations ${ }^{21}$ and IFN- $\gamma$ is a proinflammatory cytokine participated in inflammation and autoimmune disease $\mathrm{e}^{22}$. Here, two receptors for type 1 interferon (Ifnar1, Ifnar2) and a receptor for IFN- $\gamma$ (Ifngr2) were found to be depleted in bat cells (Figure S1). In summary, our results suggested that the transcriptions of some pro-inflammatory cytokine receptors were suppressed in bat lung cells, which could probably provide novel insights about the specific immune characteristics of bats.

Due to experimental limitations, we have only characterized the cytokine 
expressions of the lung cells of bats, cats, tiger and pangolins in healthy status, which may help unravel the baseline expression of immune factors. To fully understand immune responses stimulated by specific viral infections in bats, transcriptome data from appropriately controlled infection experiments are desired to better illustrate the differential pulmonary immune responses between these species. Moreover, because bats, tigers and pangolins were feral species, the animals sampled may not be strictly healthy as the pathogen-free laboratory animals. Therefore, our data might be influenced by individual bias to some extent.

In this study, we have generated the single-cell transcriptomes for the lungs of four non-model species associated with the cross-species transmissions of SARS-CoV-2. Besides, bats and animals such as cats and tigers are frequently involved in the cross-species transmissions of other viruses, such as various subtypes of influenza A viruses. Our transcriptome data revealed their cellular heterogeneity and thus laid the foundation for in-depth comparative study regarding the cellular and immune biology upon virus infections.

\section{MATERIAL AND METHODS}

\section{Ethical statement}

Sample collection and research were performed with the approval of Institutional Review Board on Ethics Committee of BGI (Approval letter reference number BGI-NO. BGI-IRB A20008). All procedures were conducted according to the guidelines of Institutional Review Board on Ethics Committee of BGI. The sampling procedures strictly followed the 'Guidelines on Ethical Treatment of Experimental Animals' established by the Ministry of Science and Technology, China.

\section{Sample collection and dissection}

Bats used in this study were all male Rhinolophus sinicus (Chinese horseshoe bat) which were identified the species by the field experts, and obtained from Guangdong province, China, then dissected and stored in $-80^{\circ} \mathrm{C}$ freezer immediately. After being isolated, the lung tissues of bats were rinsed by $1 \mathrm{X}$ PBS and stored in liquid nitrogen.

Subsequently, we used mechanical extraction method from previous study ${ }^{23}$ to obtain 
the single nucleus and then stained using DAPI (4',6-diamidino-2-phenylindole), calculated the density of the nucleus to ensure the quality of the single nucleus RNA sequencing library construction.

\section{Single cell RNA sequencing library preparation and sequencing}

The isolated nuclei were separated from lung tissue of bats and then their reactions were performed according to the manufacturer's protocol for the Chromium Single Cell 3' GEM, Library \& Gel Bead Kit v3.1. Library preparation was carried out following the guidelines provided by the manufacturer, total of 2 libraries were sequenced using a compatible Illumina NovaSeq 6000 platform.

\section{Processing of single-nucleus RNA-seq data}

Raw sequencing data was aligned to ref genome sequence of Rhinolophus sinicus (GCF_001888835.1_ASM188883v1) and preprocessed by CellRanger 3.0.2 (10X Genomics). After obtaining the single cell gene expression matrices, we used Seurat 3.2.2 $2^{24}$ to perform the downstream analysis. First, genes detected in less than three cells were discarded. Then, low-quality cells in which expressed gene numbers are less than 200 were filtered out. Moreover, cells with the percentage of mitochondrial genes more than $10 \%$ were removed. After quality control, "LogNormalize" function was used to normalize and "FindVariableGenes" was used to calculate the variance scores of each gene. Then, we applied "cca" to integrate the two libraries and remove the batch effect. Following, the integrated data was scaled and principal component analysis (PCA) was performed on the corrected data. Clusters were identified using "FindClusters" function and visualized by UMAP.

\section{Data collection}

Other single cell RNA sequencing data (cat, tiger, pangolin) were obtained from public $^{1}$.

\section{Annotation}

The lung cell types of bats were annotated according to the expression of canonical markers. The lung cell types of cat, tiger and pangolin were acquired from Chen et al.

\section{DEGs identification and GO enrichment analysis}

We applied "FindAllMarkers" function to identify the differentially expressed genes 
(DEGs). P value of the significance of DEGs were calculated by default Wilcox test and adjusted using Bonferroni methods. Genes of which adjusted p value is less than 0.05 and absolute value of log fold change is more than 0.25 were defined as DEGs and used for the following analysis. R package clusterProfiler ${ }^{25}$ was applied for GO term enrichment analysis.

\section{Cross-species data integration}

To facilitate the comparation among four species, we first convert all genes of bats to the homogenous mouse genes using OrthoFinder ${ }^{26}$. Then we used Seurat ${ }^{2}$ to integrate the single cell datasets of bats, cat, tiger and pangolin.

\section{Cell type specific expression patterns of respiratory virus receptors and cytokine}

Used the integrated datasets of four species, we calculated the average expression value and the percentage of expression for known respiratory virus receptors collected from previous study ${ }^{27}$ in various cell types. Besides, the expression patterns of cytokine genes in each cell type were also calculated using "DotPlot" function from Seurat and visualized by ggplot $2^{28}$.

\section{ACKNOWLEDGMENTS}

This work was supported by the Guangdong Major Project of Basic and Applied Basic Research (Grant 2020B0301030007), the Local Innovative and Research Teams Project of Guangdong Pearl River Talents Program (Grant 2019BT02N054), the

Program for Changjiang Scholars and Innovative Research Team in University of Ministry of Education of China (Grant No. IRT_17R39), the Innovation Team Project of Guangdong University (Grant No. 2019KCXTD001)

\section{AUTHOR CONTRIBUTION}

Conceptualization, D.C., J.S., H.L., J.Z., L.L. and X.D.; methodology, D.C., P.D., X.W., Z.O. and J.Z; software, X.W. and P.D.; validation, X.W. P.D. and Z.O.; formal analysis, X.W. and P.D.; investigation, C.S., X.W., P.D., W.W. and Y.W.; resources, M.L. D.C. and X.J.; data curation, X.W. and P.D.; writing - original draft preparation, X.W., Z.O. and P.D.; writing - review and editing, X.W., Z.O., P.D., J.S., W.Z., and 
D.C.; visualization, P.D. and X.W.; supervision, J.S., H.L. and D.C.; project administration, D.W., J.S., H.L. and D.C.; funding acquisition, J.S. D.C. and H.L.. All authors have read and agreed to the published version of the manuscript.

\section{CONFLICT OF INTEREST}

The authors declare no competing interests.

\section{DATA AVAILABILITY STATEMENT}

The raw data supporting the findings of this study will be made available upon request. The data that support the findings of this study have been deposited into CNGB Sequence Archive (CNSA) ${ }^{29}$ of China National GeneBank DataBase $(\mathrm{CNGBdb})^{30}$ with accession number CNP0002166. 


\section{FIGURE LEGENDS}

Figure 1. Comparative single cell lung atlas of the bat, cat, tiger and pangolin. a, Illustration of the overall project design. b, UMAP plot of bat lung. c. Violin plot showing the expression patterns of canonical cell type markers. d. Expression proportion and scaled expression value of virus receptors in distinct cell types of bat, cat, tiger and pangolin.

\section{SUPPLEMENTARY FIGURES AND TABLES}

Figure S1: Dot plot showing the expression percentage and scaled expression value of cytokine and their receptors in distinct cell types of bat, cat, tiger and pangolin.

Table S1: Differentially expressed genes (DEGs) of bat lung.

\section{REFERENCES}

1. Wynne, J. W. et al. Proteomics informed by transcriptomics reveals Hendra virus sensitizes bat cells to TRAIL-mediated apoptosis. Genome biology 15, 532 (2014).

2. Glennon, N. B., Jabado, O., Lo, M. K. \& Shaw, M. L. Transcriptome Profiling of the Virus-Induced Innate Immune Response in Pteropus vampyrus and Its Attenuation by Nipah Virus Interferon Antagonist Functions. Journal of Virology 89, 7550-7566 (2015).

3. Papenfuss, A. T. et al. The immune gene repertoire of an important viral reservoir, the Australian black flying fox. BMC Genomics 13, (2012).

4. Xie, J. et al. Dampened STING-Dependent Interferon Activation in Bats. Cell Host and Microbe 23, 297-301.e4 (2018).

5. Zhou, P. et al. Contraction of the type i IFN locus and unusual constitutive expression of IFN- $\alpha$ in bats. Proceedings of the National Academy of Sciences of the United States of America 113, 2696-2701 (2016). 
6. Irving, A. T., Ahn, M., Goh, G., Anderson, D. E. \& Wang, L. F. Lessons from the host defences of bats, a unique viral reservoir. Nature vol. 589 363-370 (2021).

7. Zhou, P. et al. Addendum: A pneumonia outbreak associated with a new coronavirus of probable bat origin. Nature 588, E6 (2020).

8. Wu, F. et al. A new coronavirus associated with human respiratory disease in China. Nature 579, 265-269 (2020).

9. $\mathrm{Hu}, \mathrm{B}$. et al. Discovery of a rich gene pool of bat SARS-related coronaviruses provides new insights into the origin of SARS coronavirus. PLOS Pathogens 13, e1006698 (2017).

10. Ge, X.-Y. et al. Isolation and characterization of a bat SARS-like coronavirus that uses the ACE2 receptor. Nature 503, 535-538 (2013).

11. Li, W. et al. Bats Are Natural Reservoirs of SARS-Like Coronaviruses. Science 310, 676-679 (2005).

12. Lam, T. T.-Y. et al. Identifying SARS-CoV-2-related coronaviruses in Malayan pangolins. Nature 583, 282-285 (2020).

13. Liu, P., Chen, W. \& Chen, J.-P. Viral Metagenomics Revealed Sendai Virus and Coronavirus Infection of Malayan Pangolins (Manis javanica). Viruses 11, 979 (2019).

14. Zhang, Q. et al. A serological survey of SARS-CoV-2 in cat in Wuhan. Emerging Microbes \& Infections 9, 2013-2019 (2020).

15. Newman, A. et al. First Reported Cases of SARS-CoV-2 Infection in Companion Animals — New York, March-April 2020. MMWR. Morbidity and Mortality Weekly Report 69, 710-713 (2020).

16. McAloose, D. et al. From People to Panthera $\square$ : Natural SARS-CoV-2 Infection in Tigers and Lions at the Bronx Zoo. mBio 11, (2020).

17. Tisoncik, J. R. et al. Into the Eye of the Cytokine Storm. Microbiology and Molecular Biology Reviews 76, 16-32 (2012).

18. Banerjee, A. et al. Novel Insights Into Immune Systems of Bats. Frontiers in Immunology 11, (2020).

19. Gadient, R. A. \& Patterson, P. H. Leukemia Inhibitory Factor, Interleukin 6, and 
Other Cytokines Using the GP130 Transducing Receptor: Roles in Inflammation and Injury. STEM CELLS 17, 127-137 (1999).

20. Machiyama, T. et al. TNF receptor associated factor 5 controls oncostatin M-mediated lung inflammation. Biochemical and Biophysical Research Communications 499, 544-550 (2018).

21. Makris, S., Paulsen, M. \& Johansson, C. Type I Interferons as Regulators of Lung Inflammation. Frontiers in Immunology 8, (2017).

22. Zhang, J. Yin and yang interplay of IFN- $\gamma$ in inflammation and autoimmune disease. Journal of Clinical Investigation 117, 871-873 (2007).

23. Chen, D. et al. Single cell atlas for 11 non-model mammals, reptiles and birds. Nature Communications 12, 7083 (2021).

24. Butler, A., Hoffman, P., Smibert, P., Papalexi, E. \& Satija, R. Integrating single-cell transcriptomic data across different conditions, technologies, and species. Nature Biotechnology 36, 411-420 (2018).

25. Yu, G., Wang, L.-G., Han, Y. \& He, Q.-Y. clusterProfiler: an R Package for Comparing Biological Themes Among Gene Clusters. OMICS: A Journal of Integrative Biology 16, 284-287 (2012).

26. Emms, D. M. \& Kelly, S. OrthoFinder: phylogenetic orthology inference for comparative genomics. Genome Biology 20, 238 (2019).

27. Travaglini, K. J. et al. A molecular cell atlas of the human lung from single-cell RNA sequencing. Nature 587, 619-625 (2020).

28. Wickham, H. ggplot2. (Springer International Publishing, 2016). doi:10.1007/978-3-319-24277-4.

29. Guo, X. et al. CNSA: a data repository for archiving omics data. Database 2020, (2020).

30. Chen, F. et al. CNGBdb: China National GeneBank DataBase. https://db.cngb.org/. 


\section{Figure 1}

a

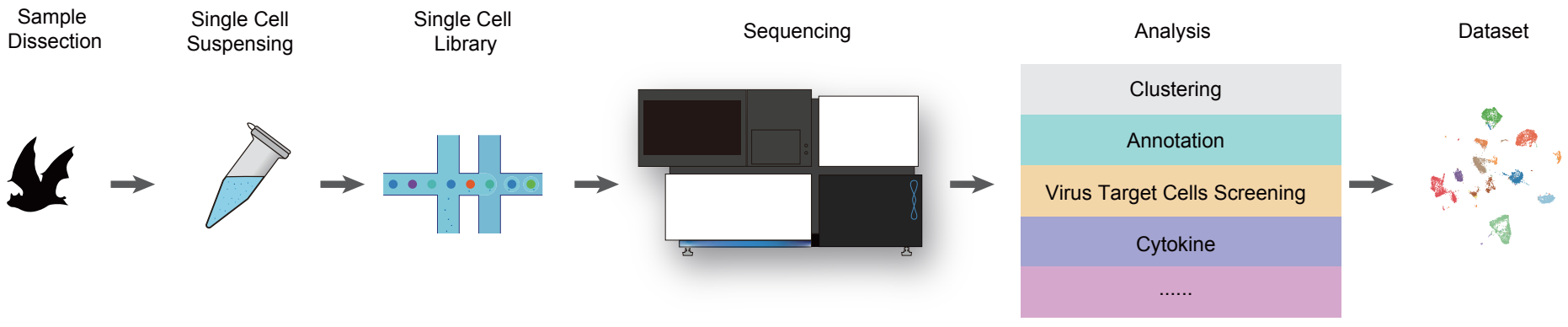

b

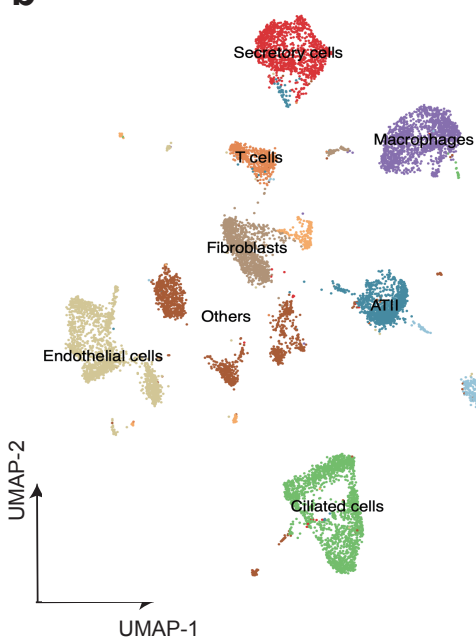

- AT1

- AT2

- Ciliated cells

- Fibroblasts

- Secrectory cells

- B cells

- T cells

- Macrophages

- Endothelial cells

- Others
C

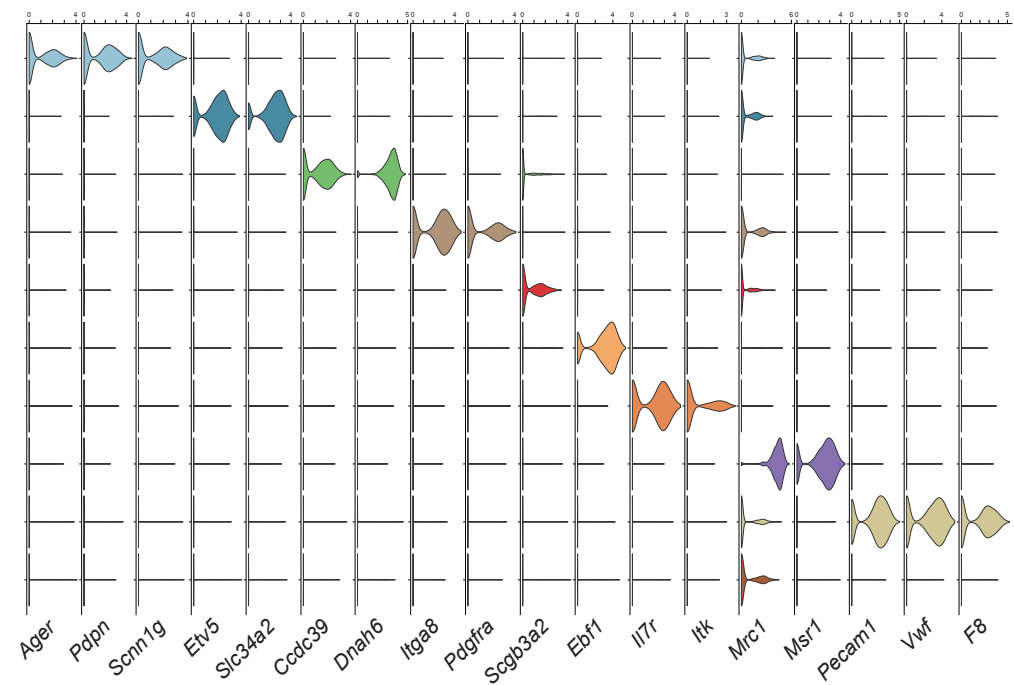

d

\begin{tabular}{|c|c|c|c|c|c|c|c|c|c|c|c|}
\hline & AT1 & AT2 & Ciliated cells & Secretory cells & Fibroblasts & Endothelial cells & Macrophages & $B$ cells & T cells & & \\
\hline AAV_2/AAV_3/AAV_8/AAV_9 & $0 \quad 000$ & 0 & 00 & $\mathrm{O}$ & $0 \quad 00$ & 00 & 0 & $0-0$ & $0 \quad 0$ & psa & \\
\hline $\mathrm{Ad}$ & $0 \begin{array}{llll}0 & 0 & 0 & 0 \\
\end{array}$ & $\begin{array}{|llll|}0 & 0 & 0 & 0 \\
\end{array}$ & 00000 & $\begin{array}{|llll|}0 & 0 & 0 & 0 \\
\end{array}$ & \begin{tabular}{|llll}
0 & 0 & 0 & 0 \\
\end{tabular} & $\begin{array}{|llll|}0 & 0 & 0 & 0 \\
\end{array}$ & $0 \begin{array}{llll}0 & 0 & 0 & C\end{array}$ & 000 & $0 \quad 0 \quad 0$ & Vwp2 & \\
\hline Ad_3/Ad_B2/MeaV & 000 & 000 & $0 \mathrm{C}$ & $0 C$ & $\circ \bigcirc 0$ & 000 & 00 & 000 & $\circ \bigcirc 0$ & $2 d 46$ & \\
\hline Ad_B & $\begin{array}{|llll|}0 & 0 & 0 & 0 \\
0 & 0 & 0 & 0 \\
\end{array}$ & \begin{tabular}{|llll}
0 & 0 & 0 & 0 \\
0 & 0 & 0 & 0 \\
\end{tabular} & $\begin{array}{|llll|}0 & 0 & 0 & 0 \\
0 & 0 & 0 & 0 \\
\end{array}$ & \begin{tabular}{|ll}
0 & 0 \\
0 & 0 \\
\end{tabular} & $\begin{array}{|llll|}0 & 0 & 0 & 0 \\
0 & 0 & 0 & 0 \\
0\end{array}$ & $\begin{array}{|llll|}0 & 0 & 0 & 0 \\
0 & 0 & 0 & 0\end{array}$ & 0 & 0 & $\begin{array}{ll}0 & 0 \\
0 & 0\end{array}$ & $\begin{array}{l}-C d 86 \\
-C d 80\end{array}$ & \\
\hline Ad_C & $\begin{array}{llll} & 0 & 0 & 0 \\
0 & 0 & 0 & 0\end{array}$ & & & $\begin{array}{ll}0 \\
0 \\
0\end{array}$ & $\begin{array}{llll}0 & 0 & 0 & 0 \\
0 & 0 & 0 & 0\end{array}$ & \begin{tabular}{|llll}
0 & 0 & 0 & 0 \\
0 & 0 & 0 & 0 \\
\end{tabular} & $\begin{array}{llll}0 & 0 & 0 & 0 \\
0 & 0 & 0 & 0\end{array}$ & $\begin{array}{lll}0 & & 0 \\
0 & 0 & \end{array}$ & $\begin{array}{lll}0 & 0 & 0 \\
0 & 0 & 0 \\
\end{array}$ & $\begin{array}{l}\text { - Itgb5 } \\
\text { - Cxadr }\end{array}$ & \\
\hline Ad_C/PareV_1 & 0000 & 0 & $\mathrm{O}$ & 0 & \begin{tabular}{|lllll}
0 & 0 & 0 & 0 \\
\end{tabular} & \begin{tabular}{|lllll}
0 & 0 & 0 & 0 \\
\end{tabular} & 00 & 000 & 0.00 & Itgav & \\
\hline CoV_229E & $0 \quad 0 \quad 0$ & $\begin{array}{|lll|}0 & 0 & 0 \\
\end{array}$ & $\begin{array}{|llll|}0 & 0 & 0 & 0 \\
\end{array}$ & $\begin{array}{|lll|}0 & 0 & 0 \\
\end{array}$ & $\begin{array}{|llll|}0 & 0 & 0 & 0 \\
\end{array}$ & $\begin{array}{|llll|}0 & 0 & 0 & 0 \\
\end{array}$ & \begin{tabular}{llll|}
0 & 0 & 0 & 0 \\
\end{tabular} & 0 & 0 & Anpep & \\
\hline CoV_229E/IFV & 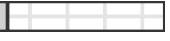 & - & L & & 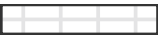 & $\square$ & 7 & 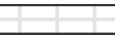 & + & Clec $4 m$ & \\
\hline CoV_MERS & $\begin{array}{llll}0 & 0 & 0 & 0 \\
\end{array}$ & \begin{tabular}{|llll}
0 & 0 & 0 \\
\end{tabular} & 0.0000 & $\begin{array}{|llll|}\circ & 0 & 0 & 0 \\
\end{array}$ & 0000 & \begin{tabular}{|llll}
0 & 0 & 0 & 0 \\
\end{tabular} & $0 \quad 0 \quad 0$ & $0 \quad 0 \quad 0$ & 000 & Dpp4 & ctexp \\
\hline CoV_NL63/SARS_CoV/SARS_CoV_2 & $\begin{array}{lllll}0 & 0 & 0 & 0 \\
\end{array}$ & $\begin{array}{|llll|}0 & 0 & 0 & 0 \\
\end{array}$ & $\begin{array}{|llll|}0 & 0 & 0 & 0 \\
\end{array}$ & \begin{tabular}{|l|l|}
0 & 0 \\
\end{tabular} & $\begin{array}{|llll|}0 & 0 & 0 & 0 \\
\end{array}$ & $\begin{array}{|lll|}0 & 0 & 0 \\
\end{array}$ & $0 \quad 0$ & 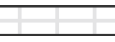 & + & Ace2 & \\
\hline HV/MetV/PareV_1 & $0 \begin{array}{lll}0 & 0 & 0 \\
\end{array}$ & $\begin{array}{|lll|}0 & 0 & 0 \\
\end{array}$ & $\begin{array}{|lll|}0 & 0 & 0 \\
\end{array}$ & $\begin{array}{|ll|}0 & 0 \\
\end{array}$ & $\begin{array}{|ll|}0 & 0 \\
\end{array}$ & $\begin{array}{|ll|}0 & 0 \\
\end{array}$ & $0 \quad 0$ & $0 \quad 0$ & $0 \quad 0$ & $\operatorname{tgb} 3$ & 75 \\
\hline IFV & +2 & $\square$ & + & \begin{tabular}{|c|c|}
+ \\
\end{tabular} & +2 & 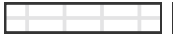 & & + & + & Gyра & \\
\hline IFV/VSV & 000 & & 0 & & 000 & 000 & $\Omega$ & 00 & 00 & Uvrag & avg.exp. \\
\hline MeaV & \begin{tabular}{|llll}
0 & 0 & 0 & 0 \\
0 & 0 & 0 &
\end{tabular} & $\begin{array}{|lll|}0 & 0 & 0 \\
0 & 0 & 0 \\
\end{array}$ & $\begin{array}{lll}0 & 0 & 0 \\
0 & 0 & 0 \\
\end{array}$ & $\begin{array}{|lll|}0 & 0 & 0 \\
0 & 0 & 0 \\
\end{array}$ & $\begin{array}{|lll|}0 & 0 & 0 \\
0 & 0 & 0 \\
\end{array}$ & $\begin{array}{|lll|}0 & 0 & 0 \\
0 & 0 & 0 \\
\end{array}$ & $\begin{array}{ll}0 & 0 \\
0 & 0 \\
\end{array}$ & $\begin{array}{ll}0 & 0 \\
0 & 0 \\
\end{array}$ & $\begin{array}{lll}0 & 0 & 0 \\
0 & 0 & 0 \\
\end{array}$ & $\begin{array}{l}\text { Slamf1 } \\
\text { Nectin4 }\end{array}$ & $\begin{array}{l}2 \\
1\end{array}$ \\
\hline MetV/ParvV_B19 & $\begin{array}{llll}0 & 0 & 0 & 0 \\
\end{array}$ & $\begin{array}{|llll|}0 & 0 & 0 & 0 \\
\end{array}$ & $\begin{array}{|llll|}0 & 0 & 0 & 0 \\
\end{array}$ & \begin{tabular}{|llll}
0 & 0 & 0 & 0 \\
\end{tabular} & $\begin{array}{|llll|}0 & 0 & 0 & 0 \\
\end{array}$ & $\begin{array}{|llll|}0 & 0 & 0 & 0 \\
\end{array}$ & $\begin{array}{llll}0 & 0 & 0 & 0 \\
\end{array}$ & 0000 & 0.00 & Itga5 & 0 \\
\hline MRV & \begin{tabular}{|lllll}
0 & 0 & 0 & 0 \\
\end{tabular} & \begin{tabular}{|lllll}
0 & 0 & 0 & 0 \\
\end{tabular} & $\begin{array}{|llll|}0 & 0 & 0 & 0 \\
\end{array}$ & \begin{tabular}{|lllll}
0 & 0 & 0 & 0 \\
\end{tabular} & $\begin{array}{|llll|}0 & 0 & 0 & 0 \\
\end{array}$ & $\begin{array}{|llll|}0 & 0 & 0 & 0 \\
\end{array}$ & 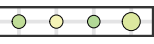 & 00 & $\begin{array}{llll}0 & 0 & 0 \\
\end{array}$ & $F 11 r$ & -1 \\
\hline MRV/ParvV_B19 & 0.0 .00 & \begin{tabular}{|lllll}
0 & 0 & 0 & 0 \\
\end{tabular} & \begin{tabular}{|lllll}
0 & 0 & 0 & 0 \\
\end{tabular} & \begin{tabular}{|lllllll}
0 & 0 & 0 & 0 \\
\end{tabular} & \begin{tabular}{|lllll}
0 & 0 & 0 & 0 \\
\end{tabular} & $\begin{array}{|llll|}0 & 0 & 0 & 0 \\
\end{array}$ & 0 & 000 & 0.00 & $\mid \operatorname{tg} b 1$ & -2 \\
\hline RhiV_A/RhiV_B & $\begin{array}{|lll|}0 & 0 & 0 \\
\end{array}$ & $\begin{array}{|ll|}0 & 0 \\
\end{array}$ & $0 \quad 0$ & \begin{tabular}{|lll}
0 & 0 \\
\end{tabular} & \begin{tabular}{|lll}
0 & 0 \\
\end{tabular} & $\begin{array}{|lll|}0 & 0 & 0 \\
\end{array}$ & 0 & 0 & 0 & Icam1 & \\
\hline RhiV_C & $\begin{array}{llll}0 & 0 & 0 & 0 \\
\end{array}$ & \begin{tabular}{|llll}
0 & 0 & 0 \\
\end{tabular} & 00 & $\begin{array}{|llll|}0 & 0 & 0 & 0 \\
\end{array}$ & $\begin{array}{|llll|}0 & 0 & 0 & 0 \\
\end{array}$ & $\begin{array}{|llll|}0 & 0 & 0 & 0 \\
\end{array}$ & $0 \quad 0 \quad 0$ & $0 \quad 0$ & 0 & Cahr3 & \\
\hline RubV & $\begin{array}{llll} & 0 & 0 & 0 \\
\end{array}$ & 0 & 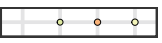 & & $\circ \quad 0 \quad 0$ & $\begin{array}{ll}0 & \circ \\
\end{array}$ & - & & 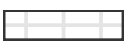 & Mog & \\
\hline SARS_CoV & $\begin{array}{ll}0 \\
0\end{array}$ & \begin{tabular}{|l|l|}
0 & 0 \\
\end{tabular} & $0 \quad 0$ & $\square$ & \begin{tabular}{|l|l}
0 \\
\end{tabular} & $\begin{array}{|ll|}0 & 0 \\
\end{array}$ & $0 \quad 0$ & 0 & 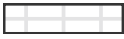 & Clec4g & \\
\hline SARS_CoV_2 & $\mid \begin{array}{llll}0 & 0 & 0 & 0 \\
0 & 0 & 0 & 0 \\
0 & 0 & 0 & 0\end{array}$ & & $\begin{array}{|llll|}0 & 0 & 0 & 0 \\
0 & 0 & 0 & 0 \\
0 & 0 & 0 & 0 \\
\end{array}$ & \begin{tabular}{|llll}
0 & 0 & 0 & 0 \\
0 & 0 & 0 & 0 \\
0 & 0 & 0 & 0 \\
\end{tabular} & \begin{tabular}{|llll}
0 & 0 & 0 & 0 \\
0 & 0 & 0 & 0 \\
0 & 0 & 0 & 0 \\
0
\end{tabular} & $\begin{array}{|llll|}0 & 0 & 0 & 0 \\
0 & 0 & 0 & 0 \\
0 & 0 & 0 & 0 \\
\end{array}$ & $\begin{array}{lll}0 & 0 & 0 \\
0 & 0 & 0 \\
0\end{array}$ & $\begin{array}{lll}0 & 0 & \\
0 & 0 & 0 \\
0 & & \\
\end{array}$ & $\begin{array}{|lll|}0 & 0 & 0 \\
0 & 0 & 0 \\
0 & 0 & 0 \\
\end{array}$ & $\begin{array}{l}\text { - Scarb1 } \\
\text { - Nrp1 } \\
-A x I\end{array}$ & \\
\hline SARS_CoV/IFV/MeaV/RSV/RVFV & 0 & \begin{tabular}{|r} 
\\
\end{tabular} & \begin{tabular}{|r} 
\\
\end{tabular} & \begin{tabular}{|r}
-0 \\
\end{tabular} & \begin{tabular}{|r} 
\\
\end{tabular} & \begin{tabular}{|l} 
\\
\end{tabular} & 0 & & 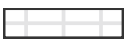 & $C d 209$ & \\
\hline VSV & \begin{tabular}{|llll}
0 & 0 & 0 & 0 \\
\end{tabular} & .0000 & 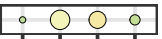 & $\circ 000$ & $\begin{array}{lllll}\circ & 0 & 0 & 0 \\
\end{array}$ & $\begin{array}{r}0 \\
\end{array}$ & 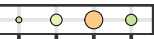 & $0 \quad 0$ & -0.0 & $L d l r$ & \\
\hline
\end{tabular}




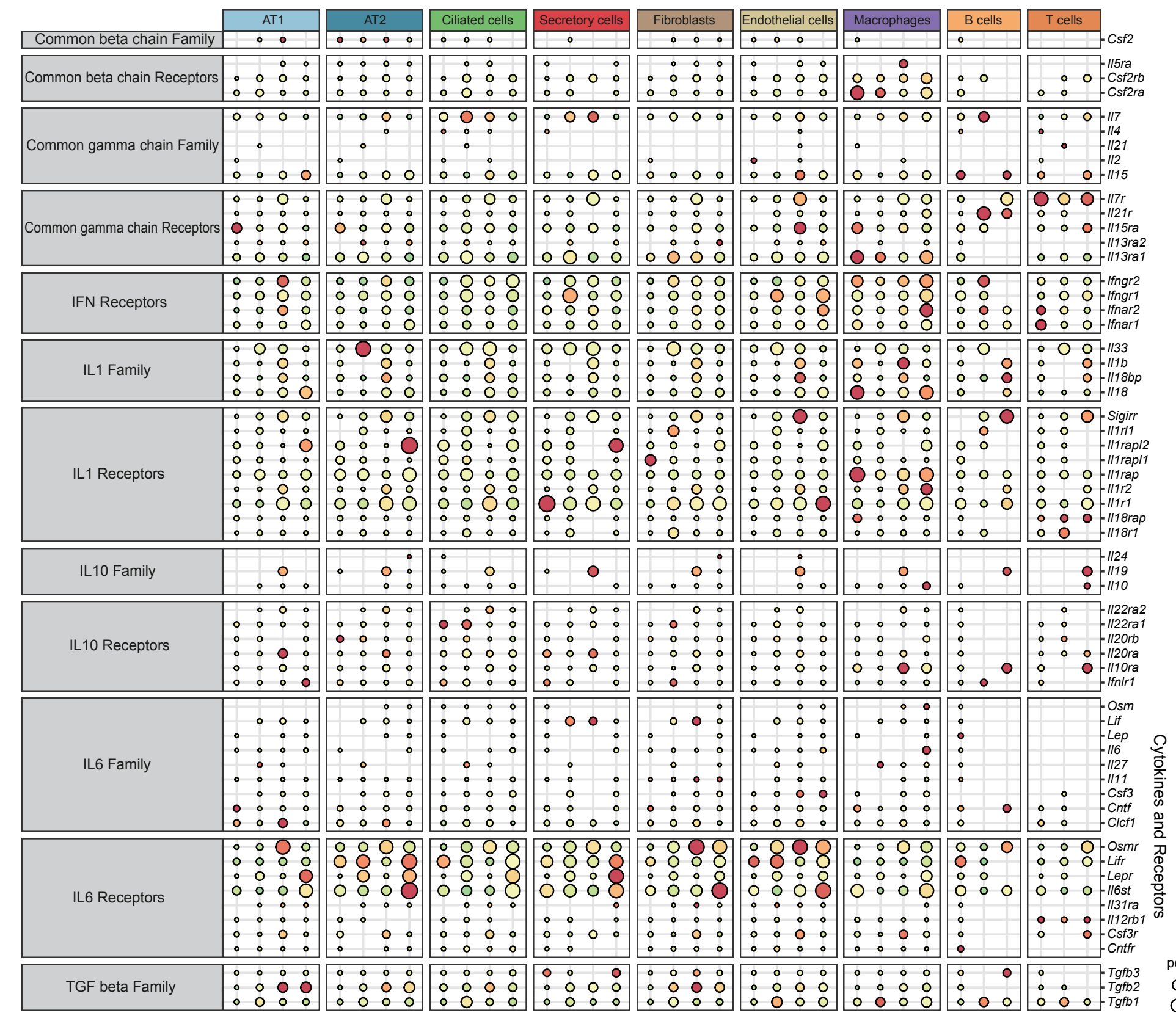

\begin{tabular}{|c|c|c|}
\hline TGF beta Family Receptors & $\begin{array}{llll}0 & 0 & 0 & 0 \\
0 & 0 & 0 & 0 \\
\circ & 0 & 0 & 0 \\
\circ & 0 & 0 & 0 \\
0 & 0 & 0 & 0 \\
0 & 0 & 0 & 0 \\
\end{array}$ & $\begin{array}{llll}0 & 0 & 0 & 0 \\
0 & 0 & 0 & 0 \\
0 & 0 & 0 & 0 \\
\circ & 0 & 0 & 0 \\
0 & 0 & 0 & 0 \\
0 & 0 & 0 & 0\end{array}$ \\
\hline TNF Family & 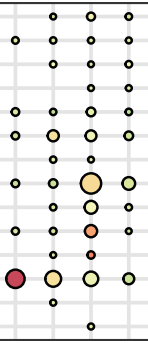 & $\begin{array}{llll} & 0 & 0 & 0 \\
0 & 0 & 0 & 0 \\
0 & 0 & & \vdots \\
0 & 0 & 0 & 0 \\
0 & 0 & 0 & 0 \\
0 & 0 & 0 & 0 \\
0 & 0 & 0 & 0 \\
0 & 0 & 0 & 0 \\
0 & 0 & 0 & \vdots \\
0 & 0 & 0 & 0 \\
0 & 0 & 0 & 0 \\
0 & 0 & 0 & 0 \\
0 & 0 & 0 & 0 \\
0 & 0 & 0 & 0\end{array}$ \\
\hline TNF Receptors & $\begin{array}{llll} & 0 & 0 & 0 \\
0 & 0 & 0 & 0 \\
0 & 0 & 0 & \\
0 & 0 & 0 & 0 \\
0 & 0 & 0 & 0 \\
0 & 0 & 0 & 0 \\
0 & 0 & 0 & 0 \\
0 & 0 & 0 & 0 \\
& 0 & 0 & 0 \\
& 0 & 0 & 0 \\
0 & 0 & 0 & 0 \\
0 & 0 & 0 & 0 \\
0 & 0 & 0 & 0 \\
0 & 0 & 0 & 0 \\
0 & 0 & 0 & 0 \\
0 & 0 & 0 & 0 \\
0 & 0 & 0 & 0 \\
0 & 0 & 0 & 0 \\
0 & 0 & 0 & 0 \\
0 & 0 & 0 & 0 \\
0 & & 0 & 0\end{array}$ & $\begin{array}{llll} & 0 & 0 & 0 \\
0 & & 0 & 0 \\
0 & 0 & 0 & 0 \\
0 & 0 & 0 & 0 \\
0 & 0 & 0 & 0 \\
0 & 0 & 0 & 0 \\
0 & 0 & 0 & 0 \\
0 & 0 & 0 & 0 \\
0 & 0 & 0 & 0 \\
0 & 0 & 0 & 0 \\
0 & 0 & 0 & 0 \\
0 & 0 & 0 & 0 \\
0 & 0 & 0 & 0 \\
0 & 0 & 0 & 0 \\
0 & 0 & 0 & 0 \\
0 & 0 & 0 & 0 \\
0 & 0 & 0 & 0 \\
0 & 0 & 0 & 0 \\
0 & 0 & 0 & 0 \\
0 & 0 & 0 & 0 \\
0 & 0 & 0 & 0\end{array}$ \\
\hline
\end{tabular}
$\left|\begin{array}{llll||llll}0 & 0 & 0 & 8 \\ 0 & 0 & 0 & 8 \\ 0 & 0 & 0 & 0 \\ 0 & 0 & 0 & 0 & 0 & 8 & 0 \\ 0 & 0 & 0 & 0 & 0 & 8 & 0 \\ 0 & 0 & 0 & 0 & 0 \\ 0 & 0 & 0 & 0 \\ 0 & 0 & 0 & 0\end{array}\right|$

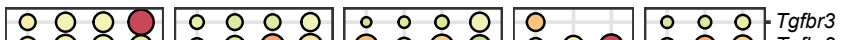
○ 75

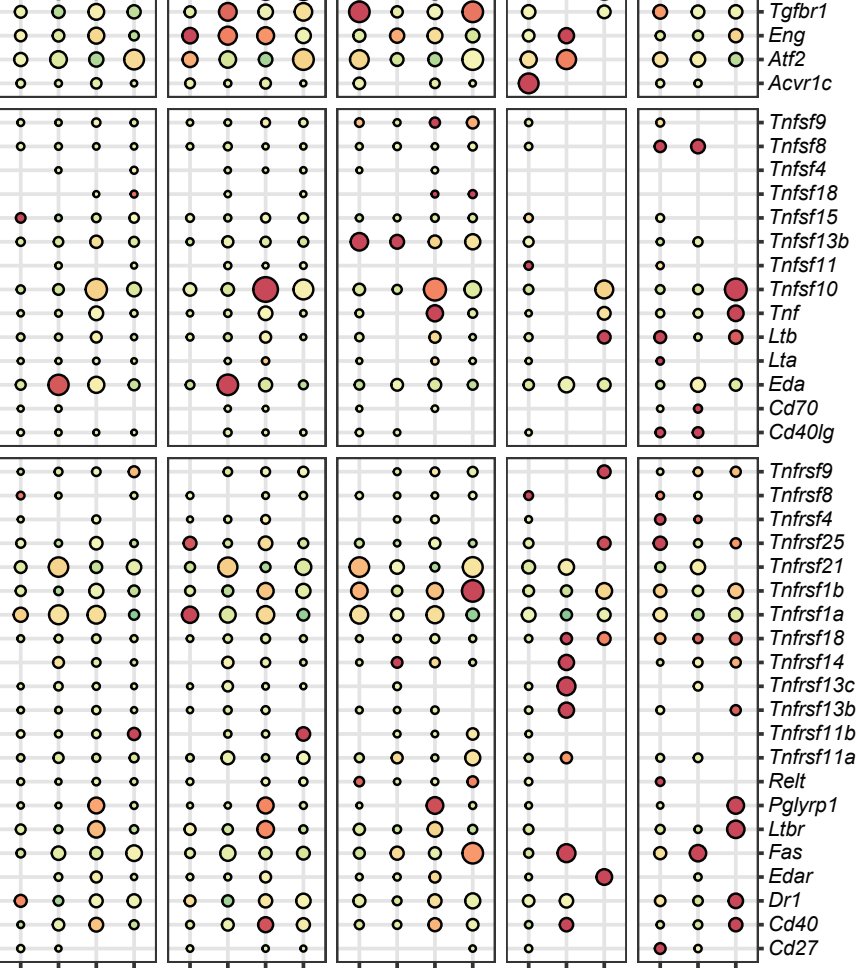
$\left.\begin{array}{llll}0 & 0 & 0 & 0 \\ 0 & 0 & 0 & 0 \\ 0 & 0 & & 0 \\ 0 & 0 & & 0 \\ 0 & 0 & 0 & 0 \\ 0 & 0 & 0 & 0 \\ 0 & 0 & 0 & 0 \\ 0 & 0 & 0 & 0 \\ 0 & 0 & 0 & 0 \\ 0 & 0 & 0 & \\ 0 & 0 & 0 & 0 \\ 0 & 0 & 0 & 0 \\ 0 & 0 & 0\end{array}\right]\left[\begin{array}{llll}0 & & & \\ 0 & 0 & 0 & 0 \\ 0 & & & 0 \\ 0 & 0 & 0 & 0 \\ 0 & 0 & & 0 \\ 0 & 0 & 0 & 0 \\ 0 & 0 & 0 & 0 \\ 0 & 0 & 0 & 0 \\ 0 & 0 & 0 & 0 \\ 0 & 0 & & 0 \\ 0 & 0 & 0 & 0\end{array}\right.$

\begin{tabular}{|llll}
0 & 0 & 0 & 0 \\
0 & 0 & 0 & 0 \\
0 & 0 & 0 & 0 \\
0 & 0 & 0 & 0 \\
0 & 0 & 0 & 0 \\
0 & 0 & 0 & 0 \\
0 & 0 & 0 & 0 \\
0 & 0 & 0 & 0 \\
0 & 0 & 0 & 0 \\
0 & 0 & 0 \\
0 & 0 & 0 & 0 \\
0 & 0 & 0 & 0 \\
0 & 0 & 0 & 0 \\
0 & 0 & 0 & 0 \\
0 & 0 & 0 & 0 \\
0 & 0 & 0 & 0 \\
0 & 0 & 0 & 0 \\
0 & 0 & 0 & 0 \\
0 & 0 & 0 & 0 \\
0 & 0 & 0 & 0 \\
0 & 0 & 0 & 0
\end{tabular}
000 $\circ 0^{\circ} 0$ $0: 000$

Species

in

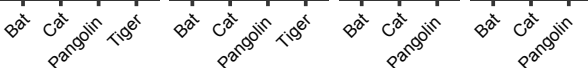

